\title{
Graphene Oxide-Polythiophene Hybrid with Broad-Band Absorption and Photocatalytic Properties
}

\author{
Shuai Wang, ${ }^{\dagger, \nabla}$ Chang Tai Nai, ${ }^{\dagger,}, \nabla$ Xiao-Fang Jiang, ${ }^{\dagger, \nabla}$ Yuanhang Pan, ${ }^{\dagger, \nabla}$ Choon-Hong Tan, \\ Milos Nesladek, ${ }^{\perp}$ Qing-Hua Xu, ${ }^{\dagger}$ and Kian Ping Loh ${ }^{*},+, \|$ \\ ${ }^{\dagger}$ Department of Chemistry, National University of Singapore, 3 Science Drive 3, Singapore 117543 \\ ${ }^{\ddagger}$ NUS Graduate School for Integrative Sciences and Engineering, National University of Singapore, Centre for Life Sciences \#05-01, \\ 28 Medical Drive, Singapore 117456 \\ ${ }^{\S}$ School of Physical \& Mathematical Sciences, Division of Chemistry and Biological Chemistry, Nanyang Technological University, 21 \\ Nanyang Link, Singapore 637371 \\ ${ }^{\perp}$ IMEC-IMOMEC, Institute for Materials Research, Hasselt University, Wetenschapspark 1, B 3590 Diepenbeek, Belgium \\ "Interdisciplinary School of Green Energy, Ulsan National Institute of Science and Technology (UNIST), Unist-gil 50, Ulsan \\ 689-798, South Korea
}

\section{Supporting Information}

ABSTRACT: Hybrid graphene oxide (GO)/poly(3-hexylthiophene-2,5-diyl) (P3HT) sheets are assembled via $\pi-\pi$ interaction and carefully isolated from the nonreacted precursors. The mutual influence of the two phases can be sharply manifested in this layer-to-layer configuration because it is undiluted by excess of one phase. To investigate the optical properties of the hybrid and possible synergistic interactions, we applied photothermal deflection spectroscopy (PDS) and pump-probe techniques. For the first time, the photocatalytic performance of these hybrids was investigated to correlate with their optical properties. The GO-P3HT hybrid demonstrates broad-band absorption and ultrafast charge transfer (1.4 ps) and acts as an excellent photocatalyst for the Mannich reaction (93\% yield).

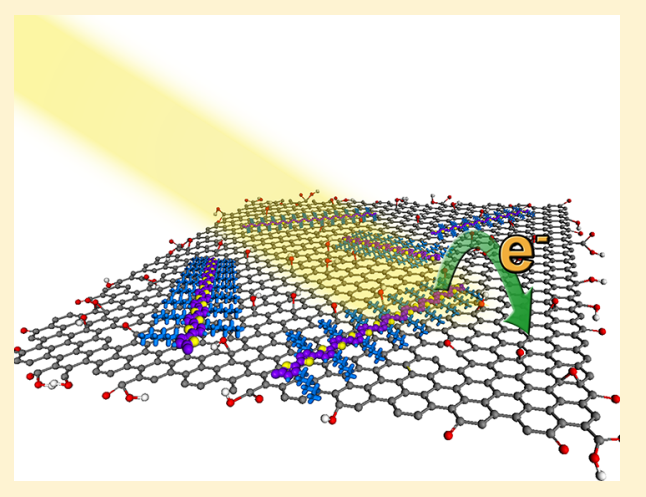

SECTION: Surfaces, Interfaces, Porous Materials, and Catalysis

$G$ O has stimulated rich interests due to its ability to mediate a multiple range of energy and charge exchange interactions with polymers, biomolecules, and organic molecules. Its structure consists of $\mathrm{sp}^{2}$ conjugated domains interspersed with polar oxygenated domains, and these properties endow GO with the ability to undergo both ionic and nonionic bonding with a wide range of molecules. For instance, polyaniline (PANI), a polymer that is often used in supercapacitors, is capable of forming composites with reduced GO (r-GO) that boasts of good conductivity, high stability, and specific capacitance, ${ }^{1}$ as well as enhanced sensitivity for hydrogen sensing. ${ }^{2}$ The GO and polyvinylpyrrolidone (PVP) composite shows high electrocatalytic activity toward the reduction of $\mathrm{O}_{2}$ and $\mathrm{H}_{2} \mathrm{O}_{2}$ and functions as a highly sensitive biosensor. ${ }^{3}$ The workfunction of $\mathrm{GO}^{4}$ falls within the band gap of polymers like $\mathrm{P} \mathrm{HT},{ }^{5}$ which suggests that $\mathrm{GO}$ can be hybridized with P3HT to form a charge-transfer complex. $\mathrm{P} 3 \mathrm{HT}$ is a semiconducting conjugated polymer that has been widely applied in organic photovoltaics and field effect transistors. r-GO has been demonstrated to improve photovoltaic performance when incorporated with $\mathrm{P} 3 \mathrm{HT}$ in solar cells. ${ }^{5-8}$ GO and P3HT can dope each other, and the extent of mutual doping depends on their respective compositions. For example, the doping of $\mathrm{P} 3 \mathrm{HT}$ by GO has been found to increase the mobility of P3HT by 20 times in the hybrid field effect transistor. ${ }^{9}$ The advantage of graphene over the CNT in organic-nanocarbon hybrids is that the composition can be tuned widely to allow for high mobility in the hybrid transistors before the device on/off ratio is affected negatively.

Various methods including direct covalent grafting, ${ }^{6}$ surfactant-assisted dispersion, ${ }^{10}$ and the chemical reduction ${ }^{11}$ of GO have been applied to facilitate the coupling between GO and P3HT. Although there are many studies on bulk composites involving excesses of either P3HT or GO in the mixtures, ${ }^{7,12}$ few studies focus on the intrinsic properties of GO that has been modified by just a thin layer of adsorbed P3HT on its aromatic scaffold. By virtue of the conjugated nature of $\mathrm{GO}$, a very thin monolayer of P3HT is expected to assemble through $\pi-\pi$ interaction between the nonpolar regions of GO and molecular layers of P3HT. The influence of either phase on

Received: July 12, 2012

Accepted: August 6, 2012

Published: August 6, 2012 
each other can be sharply manifested in this layer-to-layer configuration because it is not diluted by a nonstoichiometric excess of one phase. To investigate the optical properties of the hybrid and the possibility of synergistic interactions arising from enhanced light harvesting and charge transfer in photocatalysis, we applied photothermal deflection spectroscopy (PDS) as well as pump-probe techniques to study charge transfer and photoinduced dynamics in these hybrids. The photocatalytic performance of these hybrids was finally investigated to see if correlation exists with their optical properties.

GO and r-GO sheets were synthesized using the reported method. ${ }^{13}$ In the synthesis of nanohybrids, GO and r-GO were first dispersed in water and DMF, respectively, through sonication at room temperature for $30 \mathrm{~min}(50 / 60 \mathrm{~Hz})$ to get single-sheet dispersion. Water and DMF were then removed by solvent extraction, and chlorobenzene was used as the solvent for hybrid synthesis. After addition of P3HT and sonication for some time, homogeneous dispersions could be obtained. The color of the GO dispersion changed from brown to light purple after hybrid formation. During purification, $r$ GO-P3HT and GO-P3HT showed markedly different dispersion properties in chlorobenzene. In contrast to the GO-P3HT, the r-GO-P3HT hybrid could form a stable dispersion in chlorobenzene even after prolonged centrifugation at $14000 \mathrm{rpm}$. The excellent dispersion of r-GO-P3HT in chlorobenzene implies that the r-GO-P3HT hybrid is more hydrophobic than GO-P3HT. Toluene was used as the poor solvent to precipitate the r-GO-P3HT sample for its purification.

Insights into structural features were obtained by atomic force microscopy (AFM) and electron microscopy. Figure S2a (Supporting Information) shows the topography of monolayer GO sheets. The cross-sectional profile of the sheet reveals that it has an average thickness of approximately $0.8 \mathrm{~nm}$. On the other hand, P3HT forms self-assembled monolayer domains on HOPG substrates with a thickness of 2-3 $\AA$ (Figure S2b, Supporting Information). The topography image demonstrates an increase in roughness on the surface of the GO sheets after hybrid formation. The cross-sectional profile serves to further illustrate this change, clearly showing an increase in the peak-topeak height on the surface. In addition, there is a small increase in the average thickness of the sheet by approximately $0.9 \mathrm{~nm}$. These differences point to the presence of a thin layer of $\mathrm{P} 3 \mathrm{HT}$ molecules deposited onto the rough surface of GO.

TEM and SEM images of hybrids show that hybrids can be well-dispersed in solutions as single sheets (Figures S4-S6, Supporting Information). The GO samples that we synthesized have sizes of around $1 \mu \mathrm{m}$, while r-GO sheets are generally smaller in size due to the introduction of defects by chemical reduction. Energy-disperse X-ray (EDX) analysis verified the existence of sulfur, arising from the P3HT chains in both samples (Figure S6, Supporting Information). TGA spectra (Figure S7, Supporting Information) proved that both GOP3HT and r-GO-P3HT show significant mass loss at $500{ }^{\circ} \mathrm{C}$, which is characteristic of the decomposition of $\mathrm{P} 3 \mathrm{HT}$; on the basis of the TGA spectra, a total coverage of 28.4 and $28.9 \%$ of P3HT is estimated in the GO-P3HT and r-GO-P3HT hybrids, respectively. The presence of $\mathrm{P} 3 \mathrm{HT}$ in hybrids was also demonstrated by the appearance of aliphatic $\mathrm{C}-\mathrm{H}$ stretching and aromatic $\mathrm{C}-\mathrm{H}$ bending of $\mathrm{P} 3 \mathrm{HT}$ in their Fourier transform infrared (FTIR) spectra (Figure S8, Supporting Information).
The photophysical properties of hybrids were explored by absorption (ABS), photothermal desorption spectroscopy (PDS), and steady-state photoluminescence (PL) studies. Figure 1 shows the PDS plot of $\mathrm{P} 3 \mathrm{HT}, \mathrm{GO}, \mathrm{r}-\mathrm{GO}$, and their (a)

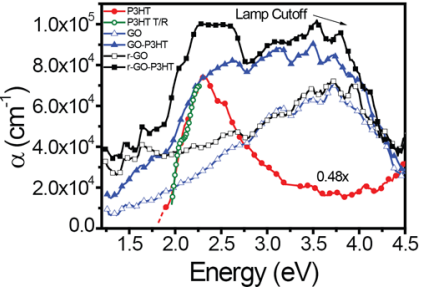

(b)

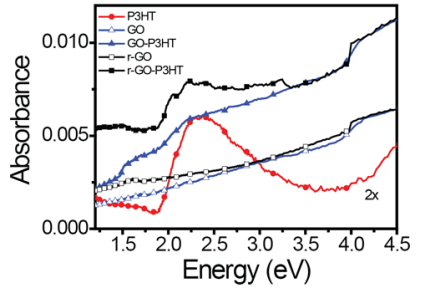

Figure 1. Plot of the absorption coefficient against the excitation energy measured by PDS (a) and UV-visible absorption spectra (b) of the same P3HT, GO, GO-P3HT, r-GO, and r-GO-P3HT films. P3HT T/R is the curve used for calibration of the absorption coefficients in PDS (see the Supporting Information). The P3HT spectra in both figures have been adjusted for ease of comparison of peak position with the composites. The absorbance curves in (b) have been normalized for comparison.

respective hybrid films as well as the UV-visible absorption (ABS) of the same films. PDS is a highly sensitive absorption technique that can be used to probe sub-band gap absorption that is usually too weak for normal absorbance techniques to detect. ${ }^{14}$ It is based on quantitative measurement of the heat energy generated by nonradiative deactivation after a sample film has been excited by a pump beam.

The PDS reveals that the GO-P3HT hybrid has broad-band absorption. Beyond $3 \mathrm{eV}$, the absorption of GO-P3HT is dominated by the strong UV absorption of GO. From 2.0 to 3.0 $\mathrm{eV}$, the absorption peak of $\mathrm{P} 3 \mathrm{HT}$ dominates the spectrum of the hybrid. Finally, below $2.0 \mathrm{eV}$, even though there is a sharp drop in absorption of $\mathrm{P} 3 \mathrm{HT}$ until it falls beyond the measurement limit of the setup (dashed line), the absorption of GO-P3HT maintains a distinct shoulder at around $1.6 \mathrm{eV}$ that is not present in the absorption of GO. This weak shoulder is also vaguely visible in the $A B S$ spectrum. We attribute this to the formation of a charge-transfer complex between GO and P3HT, which creates additional sub-band gap states that results in absorption at lower energies than the band gap absorption. A similar charge-transfer state has been reported to be detected by PDS for the donor-acceptor pair, MDMO-PPV/PCBM. ${ }^{14}$ Similar to GO-P3HT, the absorption of r-GO-P3HT also demonstrates broad-band absorption, a new peak matching with the P3HT peak position, and an absorption shoulder at around $1.8 \mathrm{eV}$. The overall absorption of $\mathrm{r}-\mathrm{GO}-\mathrm{P} 3 \mathrm{HT}$ appears stronger than that of GO-P3HT, which is likely due to the inherently stronger absorption of r-GO compared to GO.

Figure $2 \mathrm{~b}$ shows that the emission of $\mathrm{P} 3 \mathrm{HT}$ is significantly quenched by GO and r-GO. The results imply that the $\pi-\pi$ interaction between P3HT molecular films and GO leads to efficient quenching of excitations due to energy exchange. To investigate the kinetics of the interaction, the fluorescence lifetime of P3HT was measured (Figure 2c). The data can be perfectly fitted by a monoexponential decay function, and no wavelength dependence of the decays was found. Compared to the lifetime of 748 ps for pristine P3HT in chloroform solution, shorter lifetimes of 532 ps for GO-P3HT and 351 ps for rGO-P3HT were observed, which means that the interaction 
(a)

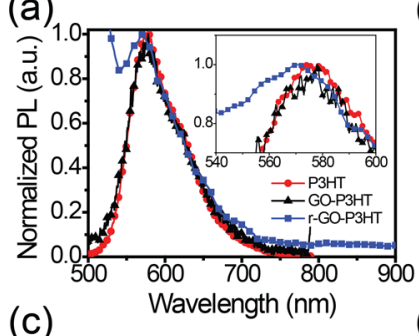

(c)

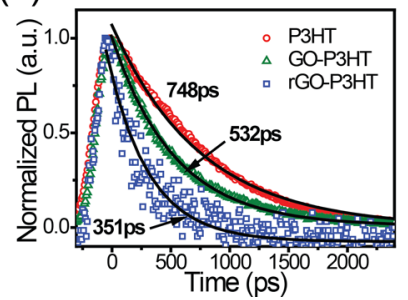

(b)

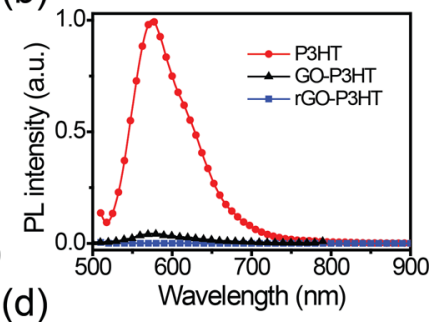

(d)

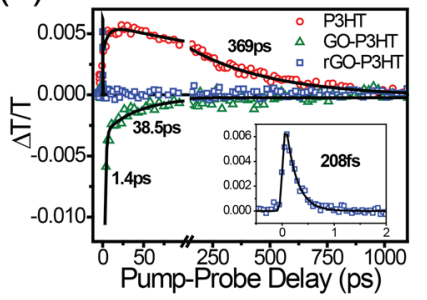

Figure 2. (a) Normalized PL spectra of a P3HT chloroform solution, $\mathrm{GO}-\mathrm{P} 3 \mathrm{HT}$ chloroform dispersion, and r-GO-P3HT chloroform dispersion. The inset shows the $540-600 \mathrm{~nm}$ range in detail. (b) PL intensity of hybrid chloroform dispersions and a P3HT chloroform solution with a concentration of $0.1 \mathrm{mg} / \mathrm{mL}$. (c) TCSPC decay curves following excitation at $400 \mathrm{~nm}$ (fluence of $46 \mathrm{~nJ} / \mathrm{cm}^{2}$ ); the emission of the P3HT sample was detected at $575 \mathrm{~nm}$. (d) Relative transmission changes for varying pump-probe delays for the $\mathrm{P} 3 \mathrm{HT}$, GO-P3HT, and $\mathrm{r}-\mathrm{GO}-\mathrm{P} 3 \mathrm{HT}$ dispersions in chloroform when excited at $400 \mathrm{~nm}$ and probed at $650 \mathrm{~nm}$. A pump fluence of $10 \mu \mathrm{J} / \mathrm{cm}^{2}$ was used. The inset shows the magnified spectrum of r-GO-P3HT in the first 2 ps.

between the P3HT and GO or r-GO accelerates the decay of emission from P3HT.

To investigate this charge-transfer process of the $\mathrm{P} 3 \mathrm{HT}$ and $\mathrm{GO} / \mathrm{r}$-GO interface, we carried out transient absorption spectra and pump-probe measurement (Figures 2d and S10, Supporting Information). Figure $2 \mathrm{~d}$ displays the transient response data traced at $650 \mathrm{~nm}$. The pristine P3HT shows positive signal for stimulated emission from excitons, with a decay time scale of $369 \mathrm{ps}$, which is consistent with the previous observations. ${ }^{15,16}$ The GO-P3HT signal shows no stimulated emission signal but a photoinduced absorption signal with two ultrafast decay time scales ( $\tau=1.4$ and $38.5 \mathrm{ps}$ ), which can be attributed to the ultrafast charge dissociation of $\mathrm{P} 3 \mathrm{HT}$ excitons at the heterointerface. ${ }^{17}$ The process can be described as such; at the interface of the GO-P3HT, the photogenerated charge pairs on the $\mathrm{P} 3 \mathrm{HT}$ are rapidly injected into $\mathrm{GO}$ within a time scale of 1.4 ps based on weak Coulombic interaction. r-GOP3HT hybrids show a positive $\Delta T$ signal with an even faster decay time $(208 \mathrm{fs})$ than epitaxial graphene $(140 \mathrm{fs}, 2 \mathrm{ps}) .{ }^{18} \mathrm{In}$ this case, after excitation, the electrons generated in P3HT are injected rapidly into r-GO. The ultrashort electron injection is in accordance with the quenched emission of GO-P3HT and r-GO-P3HT. Both GO-P3HT and r-GO-P3HT show ultrafast charge separation at around $1 \mathrm{ps}$, which is useful in solar energy conversion application. ${ }^{19}$

One promising application to take advantage of the broadband absorption and ultrafast charge transfer of the hybrid is photocatalysis. We have recently developed a cooperative catalyst system based on an organic dye and GO and applied it successfully in photocatalyzing oxidative $\mathrm{C}-\mathrm{H}$ functionalization of tertiary amines. ${ }^{20}$ The photocatalytic activities of both $\mathrm{r}$ GO-P3HT and GO-P3HT for the oxidative Mannich reaction were tested under very mild reaction conditions, using easily available $11 \mathrm{~W}$ fluorescence bulbs as the light

source (Table 1). In a recent report, the reaction was catalyzed by $\mathrm{Ru}(\mathrm{bpy})_{3} \mathrm{Cl}$ under visible light irradiation. ${ }^{21}$ The results

Table 1. Oxidative Mannich Reaction between $\mathrm{N}$-Aryletrahedroisoquinoline and Acetone ${ }^{a}$

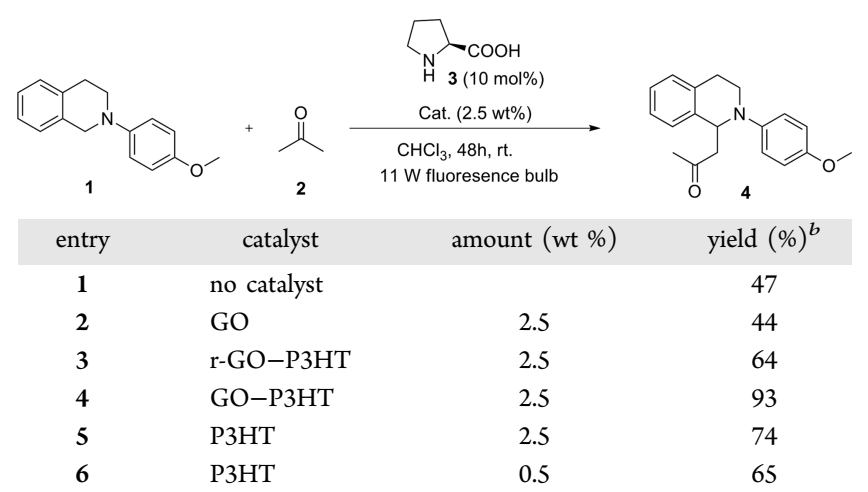

${ }^{a}$ The reaction was performed using $0.1 \mathrm{mmol}$ of $\mathbf{1}$ in the mixed solvent of $0.5 \mathrm{~mL}$ of acetone and $0.5 \mathrm{~mL}$ of $\mathrm{CHCl}_{3} .{ }^{b}$ Isolated yield.

show that $\mathrm{r}-\mathrm{GO}-\mathrm{P} 3 \mathrm{HT}$ improves the reaction yield from 40 to $60 \%$, but GO-P3HT shows even a higher conversion (93\%) compared to r-GO-P3HT. The catalytic yield of GO-P3HT is quite impressive when one takes into account that it contains only about $28-29 \%$ P3HT by weight according to TGA analysis. Weight for weight, a comparable amount of P3HT as the catalyst could only achieve a $65 \%$ conversion efficiency. Despite the fact that both composites demonstrate similar broad-band absorption, GO-P3HT shows a higher catalytic yield, indicating that besides broad-band absorption, other factors, such as charge separation and hydrophilic groups on GO, are also involved. The high catalysis performance should be due to the synergistic effect of GO and P3HT.

The reaction is initiated by photoexcited electron and hole pairs of P3HT (Figure S11, Supporting Information). A suggested mechanism is this: the tertiary amine is oxidized by the positive hole on the HOMO of $\mathrm{P} 3 \mathrm{HT}$ via single electron transfer to form the radical cation. At the same time, the excited electron is injected from the LUMO of P3HT into GO films, which is then used to activate molecular oxygen to form the dioxygen radical anion, which can be stabilized by the aromatic scaffold in GO. Mechanistically, the tertiary amine radical cation donates one hydrogen atom to the dioxygen radical anion and results in the formation of an iminium, which participates in the Mannich reaction as an acceptor via the enamine mechanism to release the final product. In addition, the oxygenated groups in the GO surface may undergo electrostatic interaction with amine radical cations, facilitating the reaction of the dioxygen radical anion to form iminium. The smaller population of oxygenated species on its scaffold may be one reason why $\mathrm{r}-\mathrm{GO}-\mathrm{P} 3 \mathrm{HT}$ shows lower catalytic activity compared to GO-P3HT. Under sunlight, the reaction can be finished in as short as $3 \mathrm{~h}$ with GO-P3HT as the catalyst (Scheme 1).

In summary, a very simple route to synthesize GO-P3HT and $\mathrm{r}-\mathrm{GO}-\mathrm{P} 3 \mathrm{HT}$ hybrids via the noncovalent binding of P3HT molecular films on GO and r-GO sheets was demonstrated. PDS, steady-state, and time-resolved PL emission spectra, as well as transient absorption spectra of hybrids, suggested picosecond charge transfer from P3HT to graphene materials. Synergistic interactions in this molecular layer-on-GO assembly enhances optical absorption, charge 
Scheme 1. Oxidative Mannich Reaction under Ambient Sunlight

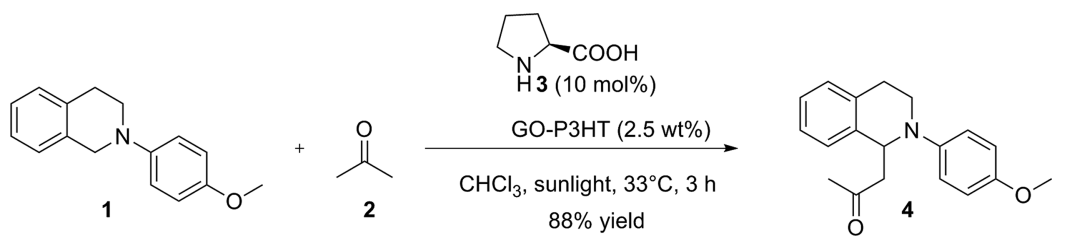

transfer, and photocatalytic properties. Due to its ability to be excited by sunlight, GO-P3HT hybrids show potential application as an efficient, green photocatalyst in organic synthesis.

\section{EXPERIMENTAL SECTION}

Synthesis of GO-P3HT. A volume of $6 \mathrm{~mL}$ of $0.4 \mathrm{mg} / \mathrm{mL} \mathrm{GO}$ aqueous dispersion was transferred to methanol and then chlorobenzene through centrifugation, followed by sonication in a bath sonicator $(50 / 60 \mathrm{~Hz})$. After the addition of $3.2 \mathrm{mg}$ of $\mathrm{P} 3 \mathrm{HT}$ and $60 \mathrm{~min}$ of sonciation at room temperature (RT), homogeneous dispersion could be obtained. A GO-P3HT and unreacted P3HT mixture was centrifuged at $10000 \mathrm{rpm}$ for 2 min to collect precipitated GO-P3HT and remove free P3HT. GO-P3HT material was then dispersed in chloroform for further applications.

Synthesis of Soluble $r$-GO-P3HT. r-GO $(2.4 \mathrm{mg})$ was added into $6 \mathrm{~mL}$ of DMF in a $10 \mathrm{~mL}$ vial and sonicated in a bath sonicator at RT for $30 \mathrm{~min}$ to get single-sheet dispersion. The DMF was then removed by a solvent extraction where toluene was added to a $2: 1$ volume ratio, and $6 \mathrm{~mL}$ of chlorobenzene was used as the solvent for hybrid synthesis. After that, $3.2 \mathrm{mg}$ of P3HT was added into the r-GO chlorobenzene dispersion and sonicated at RT for $30 \mathrm{~min}$. The product was then washed with chlorobenzene/toluene with a 1:2 volume ratio through centrifugation at $14000 \mathrm{rpm}$ for $30 \mathrm{~min}$ to remove excess polymer and finally dissolved in chloroform.

Oxidative Mannich Reaction between N-Aryl-tetrahydroisoquinoline and Acetone. (Table 1) 1 (23.9 mg, $0.1 \mathrm{mmol}, 1.0$ equiv) was added to a dispersion of GO-P3HT $(0.6 \mathrm{mg}, 2.5 \mathrm{wt} \%)$ in the mixed solvent of $0.5 \mathrm{~mL}$ of acetone, 2 , and $0.5 \mathrm{~mL}$ of $\mathrm{CHCl}_{3}$, followed by addition of L-proline $(1.2 \mathrm{mg}, 0.01 \mathrm{mmol}$, $10 \mathrm{~mol} \%)$. The reaction mixture was stirred under $11 \mathrm{~W}$ fluoresence bulb irradiation at RT. After $48 \mathrm{~h}$, the solvent was removed in vacuo, and the crude product was directly loaded onto a short silica gel column. Flash chromatography was performed using gradient elution with hexane/EA mixtures (40/1-10/1 ratio). After removing solvent, product 4 (27.5 $\mathrm{mg}$ ) was obtained as a yellow oil in $93 \%$ yield.

\section{ASSOCIATED CONTENT}

\section{S Supporting Information}

Further experimental details, instrumentation, reaction schematic, AFM of GO and GO-P3HT hybrid, SEM, TEM, TGA, and FTIR of GO-P3HT and r-GO-P3HT hybrids, time delay dependence of difference transmittance of P3HT and both hybrids, and schematic of proposed mechanism in catalyzing the Mannich reaction. This material is available free of charge via the Internet at http://pubs.acs.org.

\section{AUTHOR INFORMATION}

\section{Corresponding Author}

*E-mail: chmlokp@nus.edu.sg.

\section{Author Contributions}

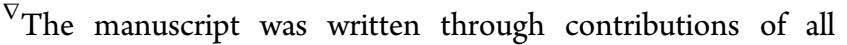
authors. All authors have given approval to the final version of the manuscript. These authors contributed equally.

\section{Notes}

The authors declare no competing financial interest.

\section{ACKNOWLEDGMENTS}

Financial support by the NRF-CRP Grant Singapore Millennium Foundation/NUS Research Horizon Fund R-143000-417-646 and Economic Development Board (SPORE, COY-15-EWI-RCFSA/N197-1) and also the World Class University program through the National Research Foundation of Korea funded by the Ministry of Education, Science and Technology (R31-2008-000-20012-0). The financial support from NanoCore Scholarship and the Grant: National Research Foundation Singapore under its Competitive Research Program (R398-001-062-281).

\section{REFERENCES}

(1) Zhang, K.; Zhang, L. L.; Zhao, X. S.; Wu, J. Chem. Mater. 2010, 22, 1392.

(2) Al-Mashat, L.; Shin, K.; Kalantar-zadeh, K.; Plessis, J. D.; Han, S. H.; Kojima, R. W.; Kaner, R. B.; Li, D.; Gou, X.; Ippolito, S. J.; Wlodarski, W. J. Phys. Chem. C 2010, 114, 16168.

(3) Shan, C.; Yang, H.; Song, J.; Han, D.; Ivaska, A.; Niu, L. Anal. Chem. 2009, 81, 2378.

(4) Liscio, A.; Veronese, G. P.; Treossi, E.; Suriano, F.; Rossella, F.; Bellani, V.; Rizzoli, R.; Samori, P.; Palermo, V. J. Mater. Chem. 2011, 21, 2924.

(5) Yu, D.; Park, K.; Durstock, M.; Dai, L. J. Phys. Chem. Lett. 2011, 2, 1113.

(6) Yu, D.; Yang, Y.; Durstock, M.; Baek, J.-B.; Dai, L. ACS Nano 2010, 4, 5633.

(7) Liu, Q.; Liu, Z.; Zhang, X.; Yang, L.; Zhang, N.; Pan, G.; Yin, S.; Chen, Y.; Wei, J. Adv. Funct. Mater. 2009, 19, 894.

(8) Kamat, P. V. J. Phys. Chem. Lett. 2009, 1, 520.

(9) Huang, J.; Hines, D. R.; Jung, B. J.; Bronsgeest, M. S.; Tunnell, A.; Ballarotto, V.; Katz, H. E.; Fuhrer, M. S.; Williams, E. D.; Cumings, J. Org. Electron. 2011, 12, 1471.

(10) Hill, C. M.; Zhu, Y.; Pan, S. ACS Nano 2011, 5, 942.

(11) Chunder, A.; Liu, J.; Zhai, L. Macromol. Rapid Commun. 2010, 31, 380.

(12) Wang, Y.; Kurunthu, D.; Scott, G. W.; Bardeen, C. J. J. Phys. Chem. C 2010, 114, 4153.

(13) Park, S.; Ruoff, R. S. Nat. Nanotechnol. 2009, 4, 217.

(14) Goris, L.; Haenen, K.; Nesládek, M.; Wagner, P.; Vanderzande, D.; Schepper, L.; D’haen, J.; Lutsen, L.; Manca, J. J. Mater. Sci. 2005, 40, 1413.

(15) Lee, Y. H.; Yabushita, A.; Hsu, C. S.; Yang, S. H.; Iwakura, I.; Luo, C. W.; Wu, K. H.; Kobayashi, T. Chem. Phys. Lett. 2010, 498, 71.

(16) Piris, J.; Dykstra, T. E.; Bakulin, A. A.; Loosdrecht, P. H. M. v.; Knulst, W.; Trinh, M. T.; Schins, J. M.; Siebbeles, L. D. A. J. Phys. Chem. C 2009, 113, 14500.

(17) Stranks, S. D.; Weisspfennig, C.; Parkinson, P.; Johnston, M. B.; Herz, L. M.; Nicholas, R. J. Nano Lett. 2011, 11, 66. 
(18) Dawlaty, J. M.; Shivaraman, S.; Chandrashekhar, M.; Rana, F.; Spencer, M. G. Appl. Phys. Lett. 2008, 92, 042116.

(19) Tachibana, Y.; Moser, J. E.; Grätzel, M.; Klug, D. R.; Durrant, J. R. J. Phys. Chem. 1996, 100, 20056.

(20) Pan, Y.; Wang, S.; Kee, C. W.; Dubuisson, E.; Yang, Y.; Loh, K. P.; Tan, C.-H. Green Chem. 2011, 13, 3341.

(21) Rueping, M.; Vila, C.; Koenigs, R. M.; Poscharny, K.; Fabry, D. C. Chem. Commun. 2011, 47, 2360. 